completed in the hospice (19) followed by those completed in hospital (13) and then GP settings (4).

Conclusions The piece of work identified the majority of ReSPECT forms completed were of high quality and had high completion rate for clinical recommendations and resuscitation decisions. Interestingly, forms completed within a hospice setting were assessed to be of higher quality. This quality improvement process used standard reporting and benchmarking quality standards to compare across the health economy. This emphasises the need for services to use co-created tools both in terms of assessment and governance.

\section{THE USE OF OPTIFLOW THERAPY IN A SPECIALIST CANCER HOSPITAL AND IT'S IMPLICATION FOR PATIENTS RECEIVING PALLIATIVE CARE}

Jennifer Skinner, Tomasz Materski, Hannah Tutcher, Angela Halley. Royal Marsden NHS Foundation Trust

10.1136/spcare-2021-PCC.83

Background Optiflow therapy (OF) is nasal high flow Oxygen that is hospital based. Its aims are to reduce work of breathing and symptomatic breathlessness. Its use is generally initiated by critical care outreach in order to prevent admissions to critical care for ventilatory support. The concern was that OF was mainly commenced acutely without considering ramifications on achieving Preferred place of care or death (PPC/ PPD). Local and National guidance is lacking for patients who are receiving palliative care. We aimed to develop a guideline for initiating OF within the hospital.

Methods A baseline retrospective notes review was conducted. 60 adult medical in-patients who started OF on the wards between October 2018 and October 2019 were included.

Results The most common reason for commencing OF was infection (61\%), Pulmonary oedema (13.4\%), Pneumonitis (4.9\%) and Pleural effusions (4.9\%). Most patients remained on OF for less than 4 days (43 patients, 71.7\%). OF was initiated in $65 \%$ patients out of hours. Most often an MDT was used to make the decision. In $43 \%$ patients a 'ceiling of treatment' decision was documented prior to starting OF: of these $35 \%$ were for full escalation, 35\% for inotropes but not intubation and $30 \%$ for ward-based treatment only. Prior to starting OF $3 \%$ had a PPD documented. The majority of patients $65 \%$ improved on $\mathrm{OF}$ and were weaned off. However, $82 \%$ patients died within 8 (most of those within the first 4) months of initiation of OF; either whilst on OF, later in the admission when stopped OF, or following discharge.

Conclusions The results suggest that although the vast majority of patients are weaned off OF, it does signal a deteriorating patient and referral to palliative care should be initiated. A guideline is being developed to support clinical decision making around the use of OF.

\section{ASSESSING THE QUALITY OF END OF LIFE CARE IN PATIENTS DYING IN AN ACUTE HOSPITAL IN LONDON AND THE IMPACT OF THE COVID-19 PANDEMIC}

Samuel Somers Heslam, Joanna Davies. Royal Surrey County Hospital, St George's NHS Foundation Trust

10.1136/spcare-2021-PCC.84
Background This audit measures the extent to which in hospital end of life care is meeting the Five Priorities of Care for the Dying Person. 1) Recognise 2) Communicate 3) Involve 4) Support 5) Plan \& Do.

Aims

1. To assess the impact of the Covid-19 pandemic on end of life care.

2. To provide trust wide feedback in preparation for increasing rates of Covid-19.

Methods Cases were audited retrospectively using quality measures based on the 2016 National End of Life Care Audit. Thirty deaths from November/December 2019 were compared with forty deaths from April 2020 (first peak of Covid-19)

Results Median age was similar pre and during pandemic (81.5 vs 80.3 ). During the pandemic $70 \%$ of patients audited tested positive for covid 19. Pre pandemic 13\% of patients died without a Treatment Escalation Plan (TEP) compared to $2 \%$ during the pandemic. Pre pandemic 27\% of patients received active treatment in the last days of life compared $48 \%$ during the pandemic. Involvement of the patient and next of $(\mathrm{NOK})$ in decisions remained $>90 \%$ pre and during pandemic. During the pandemic spiritual/cultural needs assessments fell by $25 \%$ and discussions around place of death fell by $36 \%$.

Conclusions The increased likelihood of active treatment in the last days of life during the pandemic may reflect the rapid disease trajectory of covid-19. The consistent high level of communication with patients and NOKs reflects efforts to communicate via telephone due to visiting restrictions. TEP completion improved during the pandemic in keeping with national drives to establish ceilings of care and the transfer to electronic TEPs in the Trust during the pandemic. Prioritisation of communication and medical intervention during the pandemic may have adversely impacted holistic needs assessment. Learning points from this audit have been publicised in the Trust in preparation for the second wave of the pandemic.

\section{GOLD STANDARDS FRAMEWORK IMPLEMENTATION DUDLEY GROUP NHS FOUNDATION TRUST}

Joanne Bowen, Kate Hall, Karen Lewis. Dudley Group NHS Foundation Trust

\subsection{6/spcare-2021-PCC.85}

Gold Standards Framework (GSF) is a systematic, evidence based approach to optimise care for all patients approaching the end of life, delivered by generalist frontline care providers. There is evidence that $30 \%$ of adult hospital inpatients are in the last year of life (Clarke et al. 2014). The specialist palliative care team have completed a yearly audit to establish the percentage of adult inpatients on a particular date in June, who died during the following 12 months. The overall percentage is 33 percent of adult inpatients on the 3rd June 2018 had died within 1 year. Therefore, this data supported the published evidence and also supported the wards with the GSF implementation programme.

Over the last 2 years the wards have engaged with six workshops and developed care plans based on national documentation for Gold Standards Framework Green, Amber and Red. 